\title{
New approaches to the development of microsatellite markers in the study of Baikal sponges
}

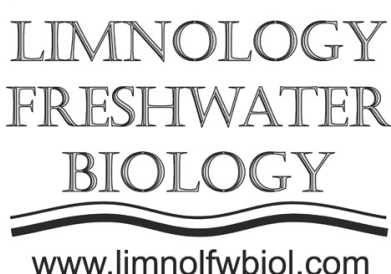

\author{
Yakhnenko A.S. ${ }^{1,2 *}$, Itskovich V.B. ${ }^{1}$ \\ ${ }^{1}$ Limnological Institute, Siberian Branch of the Russian Academy of Sciences, Ulan-Batorskaya Str., 3, Irkutsk, 664033, Russia \\ ${ }^{2}$ Joint Institute for Nuclear Research, 6 Joliot-Curie St, Dubna, Moscow Region, 141980, Russia
}

\begin{abstract}
Microsatellite markers are widely used to solve problems related to the population structure and genetic diversity of organisms. The study of the population structure of cosmopolitan freshwater sponges and endemic Baikal sponges is necessary because sponges serve as biological indicators. Population studies of Baikal endemic sponges have not been previously conducted. Due to the mass mortality and disease of sponges observed in Lake Baikal in the past decade, research in this field is of special relevance. In this work, for the first time, we developed universal microsatellite markers for population genetic studies of freshwater sponges of the species Lubomirskia baikalensis and Ephydatia muelleri using bioinformatics methods based on complete genomes analyses. In total, we developed 75 loci with microsatellite sequences of a length suitable for both species. These markers will be tested on sponge samples of the species Lubomirskia baikalensis and Ephydatia muelleri that have different life cycles and habitat conditions at early stages.
\end{abstract}

Keywords: Genetics, Baikal sponges, population genetics, microsatellite marker, evolution, ecology

\section{Introduction}

Population-genetic studies now play an important role in investigating the structure of natural communities and the anthropogenic impact, from which they suffer. Over the past decade, microsatellite markers have been widely used to solve problems related to the population structure and genetic diversity of organisms (Gui and Zhou, 2010; Vieira et al., 2016). The research on the population structure of the cosmopolitan freshwater sponges and endemic Baikal sponges is currently very relevant. In recent years, mass mortality and disease of sponges were observed in Lake Baikal, which is an alarming sign, because sponges make up the bulk of the benthos biomass in the lake. (Pile et al., 1997) According to the existing data, sponge disease can be associated with the negative impact of such factors as anthropogenic pollution, increase in water temperature, eutrophication and the introduction of alien species (Webster et al., 2008a; 2008b; Löpez-Legentil et al., 2010) as well as accompanied by a change in the microbiome of the sponges.

Additionally, sponges, being filter feeders, serve as a biomarker of the state of aquatic ecosystems. Until recently, the development of microsatellite markers has been problematic, because it was difficult to distinguish sponge genes from eukaryotic symbiont genes and required large volumes of laboratory wet work. The development of universal markers for several species was possible only by testing in the laboratory previously developed markers for closely related species. However, after the implementation of NGS sequencing methods and the publication of the first genomes, the development of microsatellite markers has become less time-consuming, which also enabled the development of microsatellite markers suitable for several species based on genomic data (Guichoux et al., 2011). In this work, for the first time, we use previously published complete genomes (Kenny et al., $2019 ; 2020$ ) for the bioinformatics-based development of universal microsatellite markers for cosmopolitan freshwater sponges, Ephydatia muellery, and endemic Baikal sponges, Lubomirskia baikalensis.

\section{Materials and methods}

Microsatellite repeats were detected in the Lubomirskia baikalensis genome with flanking regions of $200 \mathrm{bp}$ on each side using a SSRome web service developed by Mokhtar et al. (2019). The detected repeats with flanking regions were aligned to the assembly of the Ephydatia muelleri genome (Kenny et al., 2020) using the Blast + package (Camacho et al., 2009). Alignments with a length of $300 \mathrm{bp}$ or more were used for further analysis. After pairwise alignment of the same loci in genomes of Ephydatia muelleri and Lubomirskia baikalensis using the BioEdit 7.0 software

*Corresponding author.

E-mail address: yakhnenkoas@lin.irk.ru (A.S. Yakhnenko) 
package, common loci with microsatellites of suitable length were identified for both species.

\section{Results}

In the Lubomirskia baikalensis genome, we identified 6900 loci containing microsatellite sequences with a unit length from 2 to $6 \mathrm{bp}$ and several repeats from 9 to 23. Upon alignment with the Ephydatia muelleri genome, 252 candidate loci were selected, $29.7 \%$ of which had length and structure of microsatellite repeats suitable for both species.

We selected 75 most promising loci. They will be tested on samples of the Ephydatia muelleri and Lubomirskia baikalensis sponges collected at sites with different landscapes and hydrological regimes, which, presumably, form different populations. These data will allow us to conduct a comparative population analysis of cosmopolitan and endemic freshwater sponges with different reproduction patterns and different life cycles. Moreover, the obtained data will contribute to the assessment of the possible recovery of the Lubomirskia baikalensis populations, which currently faces mass disease and mortality.

\section{Acknowledgments}

We thank Morad M. Mokhtar PhD from Agriculture Genetic Engineering Research Institute, Egypt, for assistance in using SSRome service. In addition, we thank Vitushenko Julia for assistance in translation of the manuscript.

\section{Funding}

The reported study was funded by RFBR and the Government of the Irkutsk Region, project number 2044-383010, and carried out within the State Task No. 0345-2019-0002.

\section{References}

Camacho C., Coulouris G., Avagyan V. et al. 2009. BLAST + : architecture and applications. BMC Bioinformatics 10. DOI: $10.1186 / 1471-2105-10-421$

Gui J.F., Zhou L. 2010. Genetic basis and breeding application of clonal diversity and dual reproduction modes in polyploid Carassius auratus gibelio. Science China Life Sciences 53: 409-415. DOI: 10.1007/s11427-010-0092-6

GuichouxE.,LagacheL., WagnerS.etal.2011.Currenttrends in microsatellite genotyping. Molecular Ecology Resourses 11: 591-611. DOI: 10.1111/j.1755-0998.2011.03014.x

Kenny N.J., Plese B., Riesgo A. et al. 2019. Symbiosis, selection, and novelty: freshwater adaptation in the unique sponges of Lake Baikal. Molecular Biology and Evolution 36: 2462-2480. DOI: 10.1093/molbev/msz151

Kenny N.J., Francis W.R., Rivera-Vicéns R.E. et al. 2020. Tracing animal genomic evolution with the chromosomallevel assembly of the freshwater sponge Ephydatia muelleri. bioRxiv. DOI: 10.1101/2020.02.18.954784

Löpez-Legentil S., Erwin P.M., Pawlik J.R. et al. 2010. Effects of sponge bleaching on ammoniaoxi dizing Archaea: distribution and relative expression of ammonia monooxygenase genes associated with the barrel sponge Xestospongia muta. Microbial Ecology 60: 561-571. DOI: 10.1007/s00248-010-9662-1

Mokhtar M.M., Atia M.A.M. 2019. SSRome: an integrated database and pipelines for exploring microsatellites in all organisms. Nucleic Acids Resourses 47: D244-D252. DOI: 10.1093/nar/gky998

Pile A.J., Patterson M.R., Savarese M., et al. 1997. Trophic effects of sponge feeding within Lake Baikal's littoral zone: 2 . Sponge abundance, diet, feeding efficiency, and carbon flux. Limnology and Oceanography 42: 178-184. DOI: 10.4319/ lo.1997.42.1.0178

Vieira M.L.C., Santini L., Diniz A.L. et al. 2016. Microsatellite markers: what they mean and why they are so useful. Genetics and Molecular Biology 39: 312-328. DOI: 10.1590/1678-4685-gmb-2016-0027

Webster N.S., Xavier J.R., Freckelton M. et al. 2008a. Shifts in microbial and chemical patterns within the marine sponge Aplysina aerophoba during a disease outbreak. Environmental Microbiology 10: 3366-3376. DOI: 10.1111/j.1462-2920.2008.01734.x

Webster N.S., Cobb R.E., Negri A.P. 2008b. Temperature thresholds for bacterial symbiosis with a sponge. ISME Journal 2: 830-842. DOI: 10.1038/ismej.2008.42 